\title{
Evaluation of prognostic factors and survival rates in malignant feline mammary gland neoplasms
}

(10) 1003-1012

(C) The Author(s) 2015

Reprints and permissions:

sagepub.co.uk/journalsPermissions.nav DOI: $10.1177 / 1098612 \times 15610367$ jfms.com

(S)AGE

\section{Cecilia B De Campos ${ }^{1,2}$, Karine A Damasceno2, Conrado O Gamba², Ana M Ribeiro², Carla J Machado³, Gleidice E Lavalle ${ }^{4}$ and Geovanni D Cassali ${ }^{2}$}

\begin{abstract}
Objectives The aim of the study was to investigate prognostic factors in feline mammary gland neoplasms, correlating them with overall survival (OS).

Methods Fifty-six primary malignant mammary gland neoplasms and 16 metastatic lymph nodes from 37 female cats were analyzed. Clinical staging, histologic type and grade, and immunohistochemistry for Ki-67, progesterone and estrogen receptor, human epidermal growth factor receptor type 2 (HER-2), cyclooxygenase-2 (COX-2) and vascular endothelial growth factor (VEGF) were evaluated. Follow-up was performed in order to correlate prognostic factors with OS.

Results Lymph node metastasis was found in 35\% of cases. Clinical stage III, tubulopapillary carcinomas and histologic grade II cases prevailed in the study. Most neoplasms were positive for hormonal receptors, negative for HER-2 overexpression and presented VEGF overexpression. Immunoreactivity for Ki-67 $(P=0.046)$ and COX-2 $(P=0.007)$ was higher in metastases than in primary tumors. COX-2 $(P=0.089)$, HER-2 $(P=0.012)$ and histologic grade $(P=0.080)$ were correlated with OS.

Conclusions and relevance The data suggest that inhibition of ovarian hormones and COX-2 may represent a therapeutic option for malignant feline mammary gland neoplasms. When evaluating disease progression, COX-2 scores and Ki-67 index should be analyzed in primary tumors and metastases. Histologic grade, HER-2 status and COX-2 scores were found to have a direct influence on OS. Prognostic factors allow for a better understanding of disease outcome in a condition that is characterized by a poor prognosis. The present work highlights the need for further studies on endocrine therapy and COX-2 inhibitors, which could influence OS.
\end{abstract}

Accepted: 12 September 2015

\section{Introduction}

Feline mammary neoplasms are the third most frequent tumor type in the domestic cat, after hematopoietic and cutaneous neoplasms. ${ }^{1-4}$ Neutered animals are less likely to develop tumors than intact cats. ${ }^{4}$ In contrast to humans and dogs, at least $80 \%$ of all feline mammary tumors are malignant. 5,6 Ulceration, lymphatic invasion and regional or distant metastasis are common findings in the majority of malignant feline mammary gland neoplasms (MFMGN) ${ }^{4}$

Prognostic factors are clinical, pathologic and biologic features of cancer patients and their tumors that forecast clinical outcome.7 Tumor size, extent of surgery and histologic grade have been described as the most significant prognostic factors for MFMGN. ${ }^{4}$ Lymph node involvement, lymphovascular invasion, tumor size and tumor
1Department of Veterinary Clinic and Surgery, School of Agricultural and Veterinary Sciences of the Sao Paulo State University (FCAV/ UNESP) - Jaboticabal Campus, Jaboticabal, Brazil

2Laboratory of Comparative Pathology, Department of General Pathology, Federal University of Minas Gerais (UFMG), Belo Horizonte, Brazil

${ }^{3}$ Department of Preventive and Social Medicine, Faculty of Medicine, Federal University of Minas Gerais (UFMG), Belo Horizonte, Brazil

${ }^{4}$ Veterinary Hospital, Veterinary School, Federal University of Minas Gerais (UFMG), Belo Horizonte, Brazil

\section{Corresponding author:}

Geovanni Dantas Cassali DVM, MS, PhD, Laboratory of Comparative Pathology, Department of General Pathology, Biological Science Institute (ICB), Federal University of Minas Gerais (UFMG), Avenida Antônio Carlos 6627 Bloco C3 Sala 166, Pampulha, PO Box 31270-901, Belo Horizonte, Brazil

Email: geovanni.cassali@gmail.com 
grade have also been described. ${ }^{8}$ Other factors that influence disease-free interval and overall survival (OS) are clinical staging, histologic subtype, mitotic index, development of metastatic disease and location of metastatic disease. Molecular markers have also been studied for feline mammary neoplasms, including human epidermal growth factor receptor type 2 (HER-2), vascular endothelial growth factor (VEGF), cyclooxygenase (COX) enzymes, proliferative index (Ki-67), and estrogen and progesterone receptors (ER and PR). ${ }^{9-11}$

Understanding prognostic factors that relate to the biologic behavior of neoplastic diseases enables tailoring of therapeutic strategies and can increase disease-free intervals and OS. The aim of this study was to investigate and correlate the prognostic factors in MFMGN.

\section{Materials and methods}

Thirty-seven female cats admitted to the Veterinary Teaching Hospital of the Federal University of Minas Gerais (UFMG), Brazil, and diagnosed with MFMGN were evaluated in a retrospective manner. Radical mastectomy was performed as the surgical treatment. Fiftysix MFMGN and 16 metastatic regional lymph nodes samples were analyzed. Samples were obtained from the Pathology Sector of the Clinical and Surgical Department of the Veterinary School and the Laboratory of Comparative Pathology in the Institute of Biological Sciences, UFMG, Brazil.

The cases were staged according to a modified World Health Organization clinical staging system for feline mammary tumors. This system evaluates tumor size, neoplastic involvement of regional lymph nodes and presence of distant metastasis, classifying neoplasms into clinical stages I-IV. ${ }^{12,13}$

Tumor specimens were collected, fixed for $48 \mathrm{~h}$ in $10 \%$ neutral-buffered formalin solution and embedded in paraffin. Histologic sections of $4 \mu \mathrm{m}$ were obtained and stained with hematoxylin and eosin. Tumors were reviewed and reclassified according to veterinary histologic criteria by three pathologists (KAD, COG, GDC).6,14 Any discrepancies were resolved by discussion. Tumors displaying multiple morphologic patterns were classified according to the predominant neoplastic pattern.

The histologic grade of all invasive carcinomas was established according to the Nottingham system. ${ }^{15}$ This system evaluates tubule formation index, nuclear pleomorphism and mitotic count (Table 1). The histologic grade of the tumor was obtained through the sum of the scores, classified as grade I (3-5 points), grade II (6-7 points) and grade III (8-9 points).

Four micrometer histologic sections were obtained for immunohistochemical analysis. Details related to target antigen, clone, manufacturer, dilution, antigen retrieval method and incubation time are described in Table 2. Immunohistochemical procedures were identified using secondary antibodies (Advance HRP; DakoCytomation). Endogenous peroxidase activity was blocked with a solution of $3 \% \mathrm{H}_{2} \mathrm{O}_{2}$ in methyl alcohol. Reagents were applied for 30 mins manually and immunoreactivity was visualized by incubating the slides for 10 mins with diaminobenzidine (DAB Substrate System; Dakocytomation). Sections from a feline mammary carcinomas known to express Ki-67, HER-2, ER and PR; sections from a human colon carcinoma known to express COX-2; and sections from a human breast cancer known to express VEGF were used as positive controls. Negative controls were assessed using normal serum (Ultra V Block; Laboratory Vision) as the primary antibody. Immunohistochemical staining evaluation is described in Table 3.

Follow-up was obtained through the evaluation of medical records, telephone interviews with owners and clinical evaluations in order to evaluate disease evolution with possible recurrences, metastases and death. When the owner could not report the cause of death (through appropriate veterinary diagnosis) the animal was considered censored.

Table 1 Criteria evaluated for histologic grade classification

\begin{tabular}{lll} 
Criteria & & Score \\
\hline Tubule formation index & $>75 \%$ of the tumor & 1 \\
& $10-75 \%$ of the tumor & 2 \\
Nuclear pleomorphism & $<10 \%$ of the tumor & 3 \\
& Small and regular nuclei & 1 \\
& Moderate increase in size and variation of nuclei & 2 \\
Mitotic count & Marked pleomorphism with large variation in size and & 3 \\
& shape of nuclei & 1 \\
& $0-8$ mitoses/10 HPF & 2 \\
& $9-16$ mitoses/10 HPF & 3
\end{tabular}

Assignment of points was carried out using an Olympus BX-40 microscope fitted with a $\times 10$ eyepiece and a $\times 40$ objective. This gives a field area of $0.239 \mathrm{~mm}^{2}$

$\mathrm{HPF}=$ high-power field 
Table 2 Immunohistochemical analysis of malignant feline mammary gland neoplasms

\begin{tabular}{|c|c|c|c|c|}
\hline Target antigen (clone) & Manufacturer & Dilution & Antigen retrieval method & $\begin{array}{l}\text { Incubation time }(h) / \\
\text { temperature }\end{array}$ \\
\hline Ki-67 (MIB-1) & Dakocytomation & $1: 25$ & $\begin{array}{l}\text { Pressurized heat }\left(125^{\circ} \mathrm{C} / 2 \text { mins }\right) \text { with } \\
\text { citrate buffer } \mathrm{pH} 6.0^{*}\end{array}$ & 1/room temperature \\
\hline ER (1D5) & Dakocytomation & $1: 20$ & $\begin{array}{l}\text { Pressurized heat }\left(125^{\circ} \mathrm{C} / 2 \text { mins }\right) \text { with } \\
\text { EDTA buffer } \mathrm{pH} 9.0^{*}\end{array}$ & 1/room temperature \\
\hline PR (HPRA2) & Neomarkers & $1: 20$ & $\begin{array}{l}\text { Pressurized heat }\left(125^{\circ} \mathrm{C} / 2 \text { mins }\right) \text { with } \\
\text { EDTA buffer } \mathrm{pH} 9.0^{*}\end{array}$ & 1/room temperature \\
\hline HER-2 (polyclonal) & Dakocytomation & $1: 200$ & $\begin{array}{l}\text { Water bath }\left(98^{\circ} \mathrm{C} / 20 \text { mins }\right) \text { with } \\
\text { citrate buffer } \mathrm{pH} 6.0^{*}\end{array}$ & $16 / 4^{\circ} \mathrm{C}$ \\
\hline COX-2 (SP21) & Neomarkers & $1: 80$ & $\begin{array}{l}\text { Water bath }\left(98^{\circ} \mathrm{C} / 20 \text { mins }\right) \text { with } \\
\text { citrate buffer } \mathrm{pH} 6.0^{\star}\end{array}$ & 1/room temperature \\
\hline VEGF (Ab-1) & Neomarkers & $1: 200$ & No antigen retrieval & 1/room temperature \\
\hline
\end{tabular}

*DakoCytomation Target Retrieval Solution

$\mathrm{ER}$ = estrogen receptor; PR = progesterone receptor; HER-2 = human epidermal growth factor receptor type 2; COX-2 = cyclooxygenase-2; VEGF = vascular endothelial growth factor; EDTA = ethylenediaminetetraacetic acid

Table 3 Immunohistochemical evaluation for Ki-67, estrogen receptor (ER), progesterone receptor (PR), cyclooxygenase-2 (COX-2), vascular endothelial growth factor (VEGF) and human epidermal growth factor receptor type 2 (HER-2)

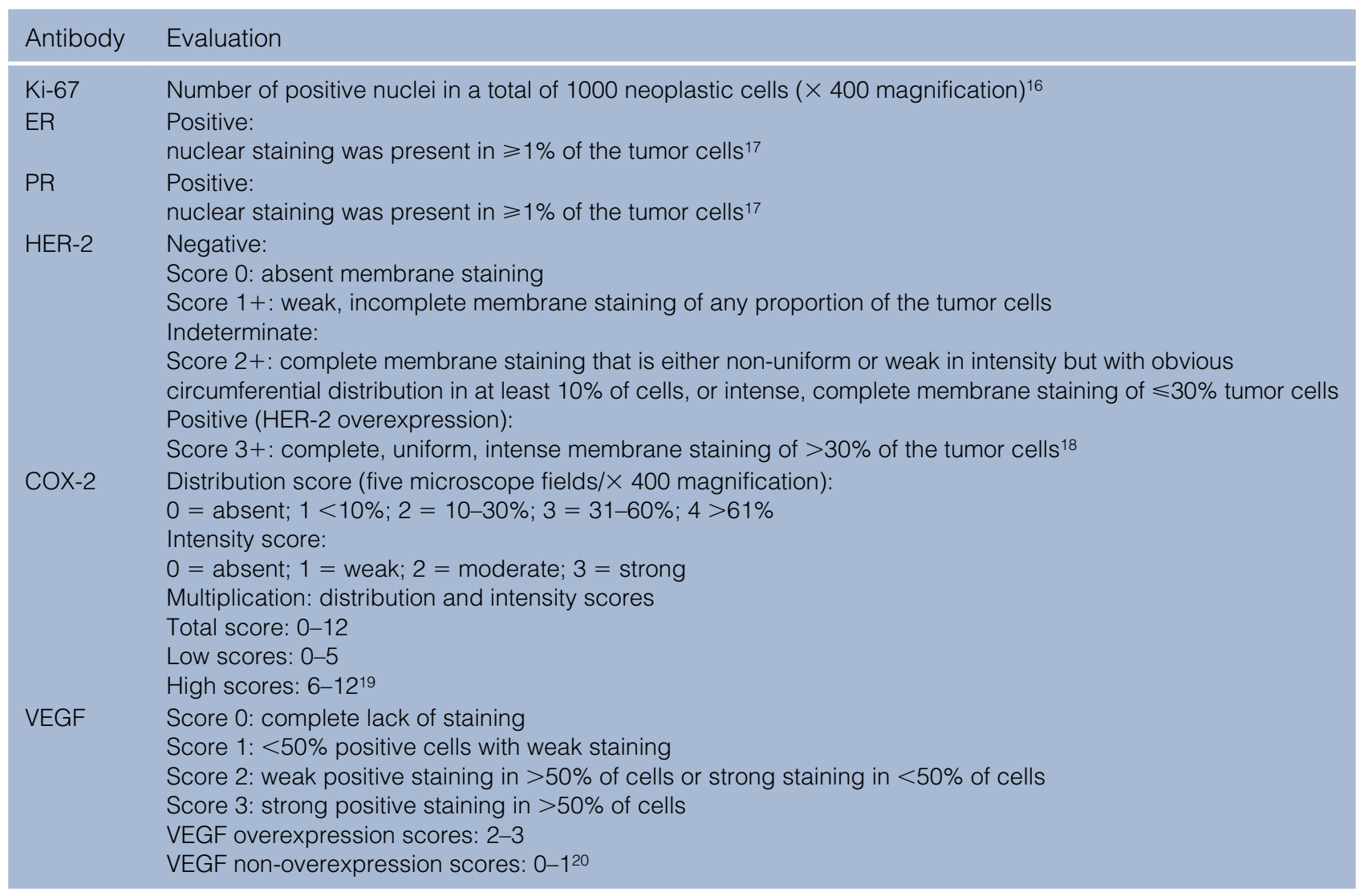

OS time was defined as the period (in days) between the date of surgical removal of the tumor and death caused by the disease. Animals that died from unknown causes or causes unrelated to the tumor were censored.
Median survival was defined as the period when $50 \%$ of the patients of a determined group died.

Statistical analyses were performed with Student's t-test, Mann-Whitney U-test and Spearman's rank 
correlation coefficient. The correlation coefficient was considered positive or negative and was classified as weak $(r \leqslant 0.35)$, moderate $(0.36 \leqslant r \leqslant 0.67)$ and strong $(\mathrm{r} \geqslant 0.68) .{ }^{21}$ OS time was evaluated by univariate KaplanMeier estimated survival curve analysis by the log-rank test (Cox-Mantel). Results were considered significant when $P \leqslant 0.05$ and trending towards significance when $P \leqslant 0.10$.

All procedures were performed under the appropriate guidelines and with the approval of the Ethics Committee for Animal Experimentation of the Federal University of Minas Gerais (CETEA/UFMG), protocol number 13412/2012.

\section{Results}

Thirty-seven queens were analyzed. Thirteen (35.13\%) patients had more than one primary neoplasm, resulting in 56 MFMGN. At the time of diagnosis, 10 (27.02\%) animals presented one, and three $(8.11 \%)$ animals presented two metastatic regional lymph nodes (axillary and/or inguinal), resulting in 16 samples of metastases (Figure 1a). Primary neoplasms were classified as six $(10.72 \%)$ carcinomas in situ and $50(89.28 \%)$ invasive malignant neoplasms.

Neoplasms were mainly diagnosed in abdominal mammary glands $(28 / 39 ; 71.80 \%)$ rather than thoracic mammary glands $(11 / 39 ; 28.20 \%)$. Skin ulceration was found in $18.60 \%$ of studied cases. Neoplasm size was classified as $<2 \mathrm{~cm}$ in $20 / 44(45.45 \%)$ cases, $2-3 \mathrm{~cm}$ in $11 / 44(25.00 \%)$ cases and $>3 \mathrm{~cm}$ in $13 / 44(29.55 \%)$ cases. Patient clinical staging demonstrated 17/25 (68.00\%) stage III, $4 / 25$ (16.00\%) stage II and 4/ $25(16.00 \%)$ stage I tumors.

The 56 primary MFMGN were diagnosed as 19 $(33.93 \%)$ tubulopapillary carcinomas (Figure 1b), 14 $(25,00 \%)$ cribriform carcinomas (Figure 1c), six (10.71\%) in situ carcinomas, four $(7.14 \%)$ papillary carcinomas, three $(5.36 \%)$ mucinous carcinomas, two $(3.58 \%)$ solid carcinomas, two (3.58\%) tubular carcinomas, two (3.58\%) glycogen-rich carcinomas, one $(1.78 \%)$ micropapillary carcinoma, one $(1.78 \%)$ carcinosarcoma, one $(1.78 \%)$ malignant adenomyoepithelioma and one $(1.78 \%)$ carcinoma in mixed tumor. Regarding histologic grade, 49 invasive carcinomas were analyzed and considered as $13(26.53 \%)$ grade I, $24(48.98 \%)$ grade II and $12(24.49 \%)$ grade III. Immunohistochemical findings are demonstrated in Table 4, and Ki-67 (Figure 1d), ER (Figure 1e), PR (Figure 1f), HER-2 score 1+ (Figure 2a) and score 3+ (Figure 2b), COX-2 low (Figure 2c) and high scores (Figure 2d), and VEGF score 1 (Figure 2e) and score 3 (Figure 2f) stainings are demonstrated.

When comparing primary tumors and metastases, statistically significant differences were observed in COX-2 and Ki-67 immunolabeling. Median COX-2 score was 3 (range 1-9) in primary tumors and 6 (range 2-12) in lymph node metastases $(P=0.007)$. Mean Ki-67 expression was $24.15 \%$ in primary tumors and $34.22 \%$ in regional metastases $(P=0.046)$.

Immunohistochemical staining for Ki-67 was weakly correlated with histologic grade $(\mathrm{r}=0.260 ; P=0.081)$, tumor size $(\mathrm{r}=0.341 ; P=0.022)$ and progressive malignant types of diagnosis (in situ carcinomas, invasive carcinomas and metastases) $(\mathrm{r}=0.322 ; P=0.007)$. HER-2 presented a weak correlation with tumor size $(r=-0.355$; $P=0.020$ ). Week correlations were also found between COX-2 and VEGF $(r=0.204 ; P=0.094)$ and COX-2 and progressive malignant types of diagnosis (in situ carcinomas, invasive carcinomas and metastases) $(r=0.359$; $P=0.002)$. Ki-67 expression in tumors with low COX-2 scores was lower $(23.07 \%)$ than in tumors with high COX-2 scores $(32.09 \%)(P=0.041)$. TNM clinical staging was weakly correlated with tumor size $(r=0.339$; $P=0.026$ ).

ER-negative tumors expressed higher HER-2 scores (median 2, range 1-3) than ER-positive tumors (median 1, range $1-3)(P=0.041)$. A significant difference was observed regarding the presence of ulceration and larger tumors (median $T_{3}$, range $T_{2}-T_{3}$ ), while smaller tumors (median $\mathrm{T}_{1}$, range $\mathrm{T}_{1}-\mathrm{T}_{3}$ ) did not present skin discontinuity $(P=0.001)$. Tumors presenting overexpression of VEGF presented higher COX-2 scores (median 4, range 1-12) than tumors that did not overexpress VEGF (median 3, range 1-8) $(P=0.019)$.

A difference trending towards significance in OS was observed when comparing high and low COX-2 scores. Patients with high COX-2 scores presented a median survival of 189 days, while low COX-2 scores were associated with a median survival of 1400 days $(P=0.089)$ (Figure 3). Regarding HER-2 expression, the median survival of cats presenting a score $1+$ was 262 days, 387 days for score $2+$ and 35 days for score $3+(P=0.012)$ (Figure 4). Histologic grade also had an impact on OS. Grade I tumors had a median survival of 1628 days, 262 days for grade II tumors and 78 days for grade III tumors $(P=0.080)$ (Figure 5).

\section{Discussion}

This study confirmed that the majority of feline mammary tumors are malignant and that there is metastasis to regional nodes at the time of diagnosis in $35.13 \%$ of the cases, similar to previously described findings, $2,6,10$ Most tumors (64\%) were classified as stage III, ${ }^{10,12}$ and there was a ratio of 7:3 abdominal to thoracic tumors. ${ }^{12}$ Ulceration of the tumors correlated to size but not to clinical stage..$^{10}$

Histologic types found in this study were diversified. Tubular, papillary, solid, cribriform and in situ carcinomas are considered common diagnosis in feline mammary glands, and some carcinomas show a combination of histologic types. ${ }^{2,4,6}$ Some histologic types found in 


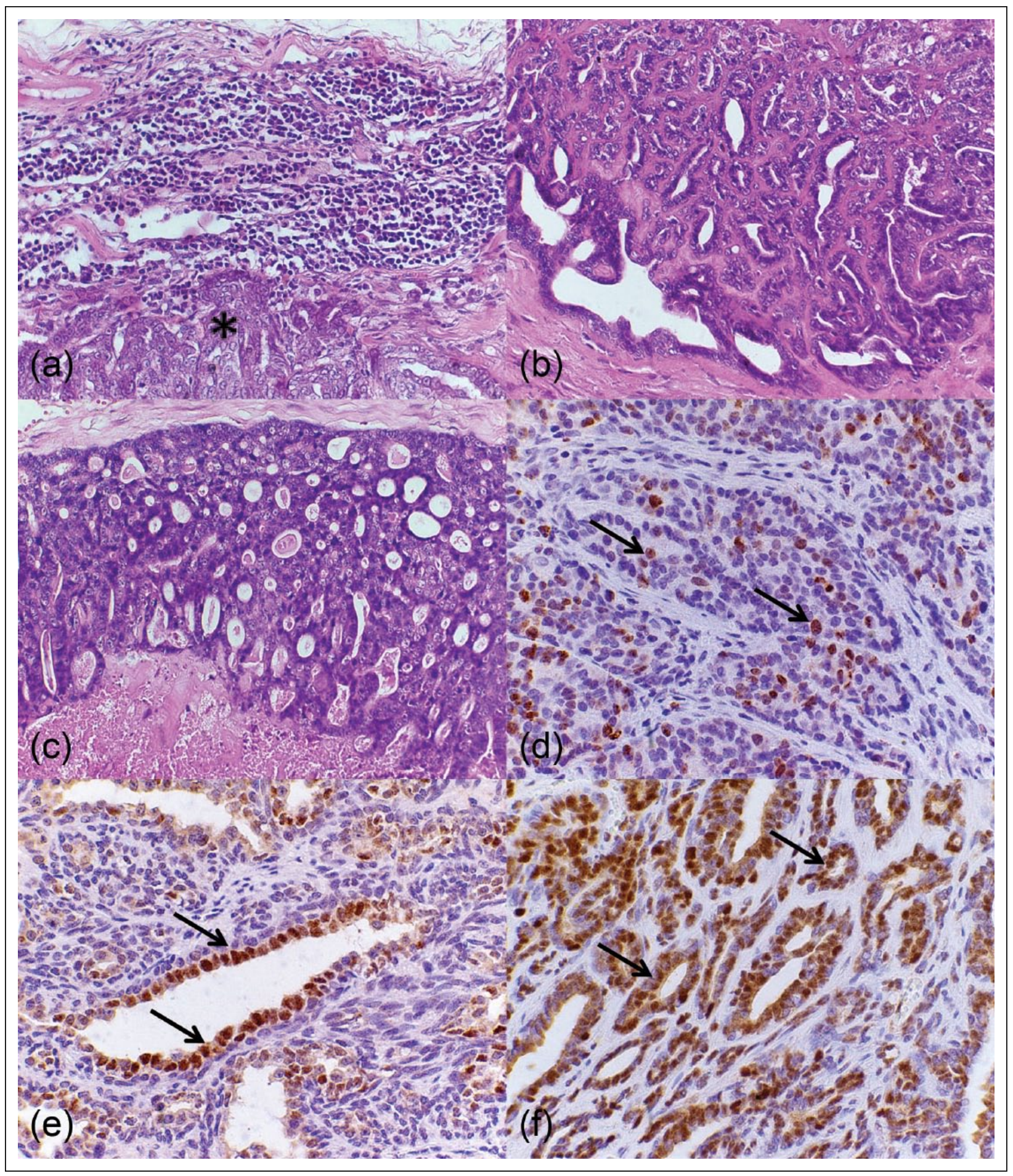

Figure 1 Histopathological and immunohistochemical analysis of malignant feline mammary gland neoplasms. (a) Feline lymph node. Regional metastasis composed of epithelial cells $\left({ }^{*}\right)$. Hematoxylin and eosin (HE), $\times 40$. (b) Feline mammary gland. Tubulopapillary carcinoma presenting an epithelial proliferation in a tubular and papillary pattern. $\mathrm{HE}, \times 40$.

(c) Feline mammary gland. Cribriform carcinoma presenting an epithelial proliferation in a cribriform pattern. HE, $\times 40$.

(d) Feline mammary gland. Tubulopapillary carcinoma presenting neoplastic Ki-67-immunoreactive epithelial cells stained in brown (nuclei) (arrows). Polymeric detection system anti-Ki-67, counterstained with Harris's hematoxylin $(\mathrm{HH}), \times 60$.

(e) Feline mammary gland. Tubulopapillary carcinoma presenting neoplastic estrogen receptor (ER)-immunoreactive epithelial cells stained in brown (nuclei) (arrows). Polymeric detection system anti-ER, counterstained with $\mathrm{HH}, \times 60$. (f) Feline mammary gland. Tubulopapillary carcinoma presenting neoplastic progesterone receptor-immunoreactive epithelial cells stained in brown (nuclei) (arrows). Polymeric detection system anti-PR, counterstained with $\mathrm{HH}, \times 60$ 


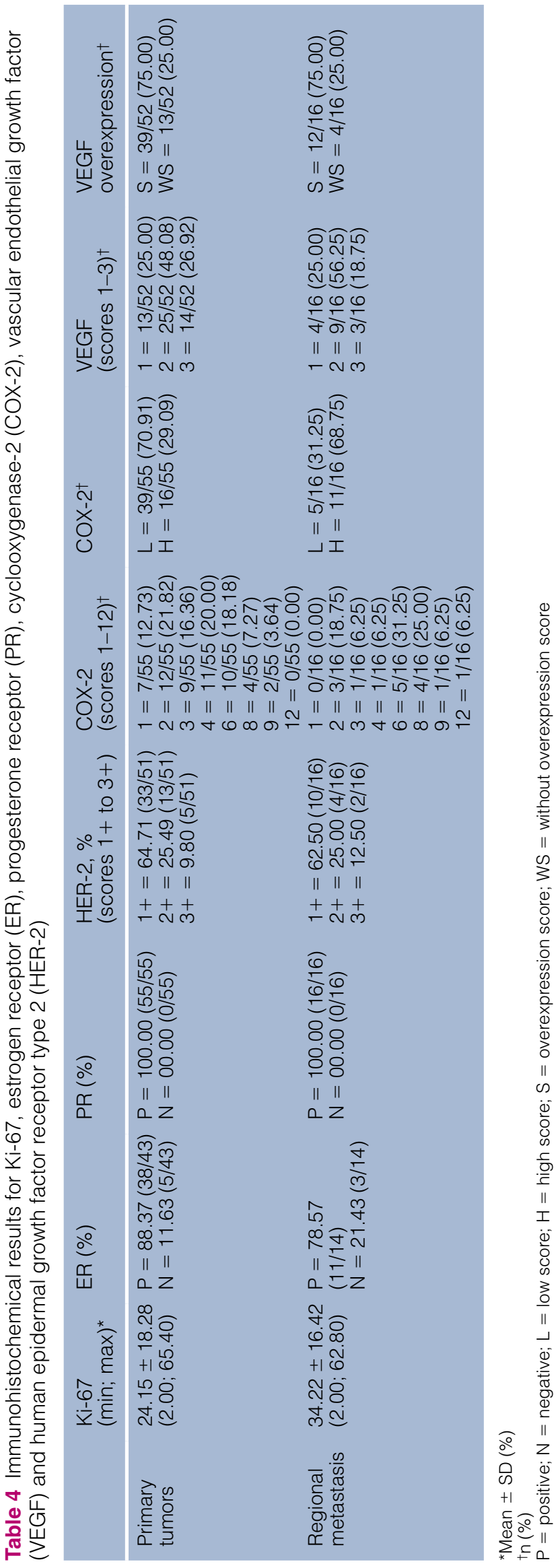

this study are described as rare in the feline mammary gland, such as mucinous carcinoma, carcinoma in mixed tumor and carcinosarcoma. 2,6

Different histologic grading methods have been developed for feline mammary tumors and further studies are needed to clarify the validity of the different grading systems for mammary carcinomas. ${ }^{22}$ The Elston and Ellis method used in this study has previously been described for MFMGN and demonstrated high prognostic value ${ }^{15}$ MFMGN are mainly classified as moderately differentiated. ${ }^{23-30}$ Our results support the prognostic value of histologic grade, described in previous studies, ${ }^{23,25}$ demonstrated by shorter OS associated with higher histologic grade. This study also demonstrated significant correlation between Ki-67 index and higher histologic grade, and, although this was not demonstrated in a previous study, ${ }^{25}$ this finding is consistent with two other studies. ${ }^{23,27} \mathrm{Ki}-67$ is an excellent marker for determining the growth fraction of a given cell population. A progressive increase in the proliferative index from normal mammary gland, non-neoplastic lesions, benign lesions, in situ carcinomas and invasive carcinomas has been documented, $8,27,31$ and this study confirms that metastatic lesions have a higher proliferative index and represent a further step in tumor progression.

In this study the American Society of Clinical Oncology and the College of American Pathologists guideline recommendations for immunohistochemical staining for ER and PR in breast cancer were used and tumors were considered positive when at least $1 \%$ of the tumor nuclei were positive. This may account for the discrepancy between these findings and prior reports, ${ }^{28,32,33}$ where the negative cut-off value was higher than $1 \%$. An additional report found $91.4 \%$ positivity for PR when a $5 \%$ cut-off value was used. ${ }^{30}$ Ovariectomy status may also influence PR expression, ${ }^{33}$ and additional studies that associate receptor expression with the reproductive status of the animal are warranted. Over-representation of mammary tumors associated with intact cats and increasing hormonal exposure, ${ }^{3}$ as well as sporadic occurrence of mammary lesions following synthetic progestins, demonstrates the role of ovarian hormones in feline mammary neoplasms. ${ }^{34}$ Endocrine therapies in women through ovarian ablation, aromatase inhibitors and tamoxifen have shown an increase in disease-free intervals and OS in endocrine responsive neoplasms. ${ }^{35}$ Similar benefit could be suggested for MFMGN as endocrine receptors are present, the main option being ovariohysterectomy.

Studies in human breast tumors have linked HER-2 amplification and overexpression of its receptor with shorter disease-free intervals and increased risk of metastasis, as well as resistance to many types of therapy. ${ }^{36}$ The majority of the HER-2 immunolabeling in this study was identified with a score of $1+$, which contradicts prior reports. ${ }^{29,31}$ Subjective interpretation and lack of 


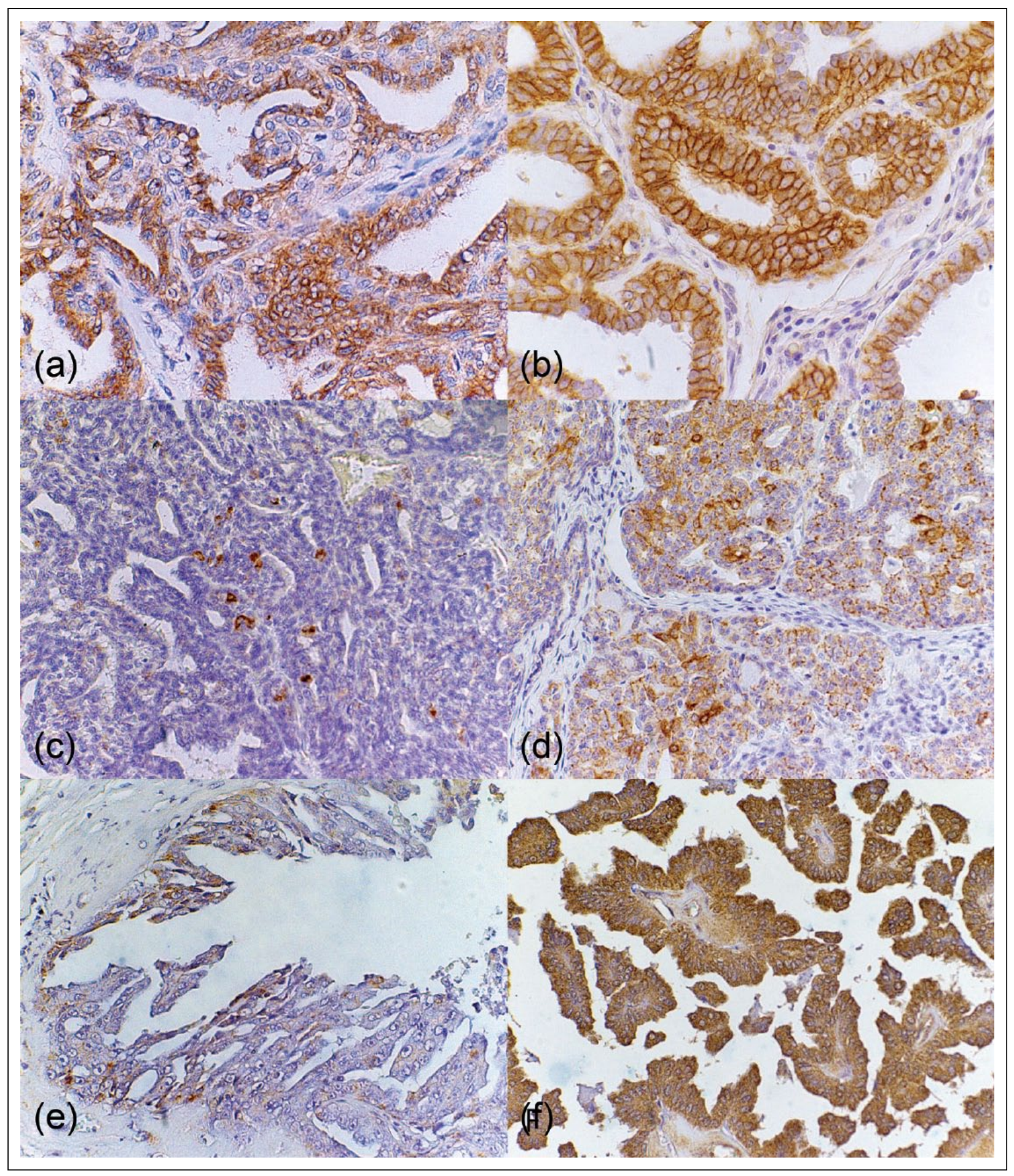

Figure 2 Immunohistochemical analysis of malignant feline mammary gland neoplasms. (a) Feline mammary gland. Tubulopapillary carcinoma presenting neoplastic human epidermal growth factor receptor type 2 (HER-2)-immunoreactive score 1+ epithelial cells stained in brown (membrane). Polymeric detection system anti-HER-2, counterstained with Harris's hematoxylin $(\mathrm{HH}), \times 40$. (b) Feline mammary gland. Tubulopapillary carcinoma presenting neoplastic HER-2-immunoreactive score 3+ epithelial cells stained in brown (membrane polymeric detection system anti-HER-2), counterstained with $\mathrm{HH}, \times 40$. (c) Feline mammary gland. Tubulopapillary carcinoma presenting neoplastic low cyclooxygenase (COX)-2-immunoreactive epithelial cells stained in brown (cytoplasm). Polymeric detection system anti-COX-2, counterstained with $\mathrm{HH}, \times 40$. (d) Feline mammary gland. Tubulopapillary carcinoma presenting neoplastic high COX-2-immunoreactive epithelial cells stained in brown (cytoplasm). Polymeric detection system anti-COX-2, counterstained with $\mathrm{HH}, \times 40$. (e) Feline mammary gland. Tubulopapillary carcinoma presenting neoplastic vascular endothelial growth factor (VEGF)-immunoreactive score 1 epithelial cells stained in brown (cytoplasm). Polymeric detection system anti-VEGF, counterstained with $\mathrm{HH}, \times 40$. (f) Feline mammary gland. Tubulopapillary carcinoma presenting neoplastic VEGF-immunoreactive score 3 epithelial cells stained in brown (cytoplasm). Polymeric detection system anti-VEGF, counterstained with $\mathrm{HH}, \times 40$ 


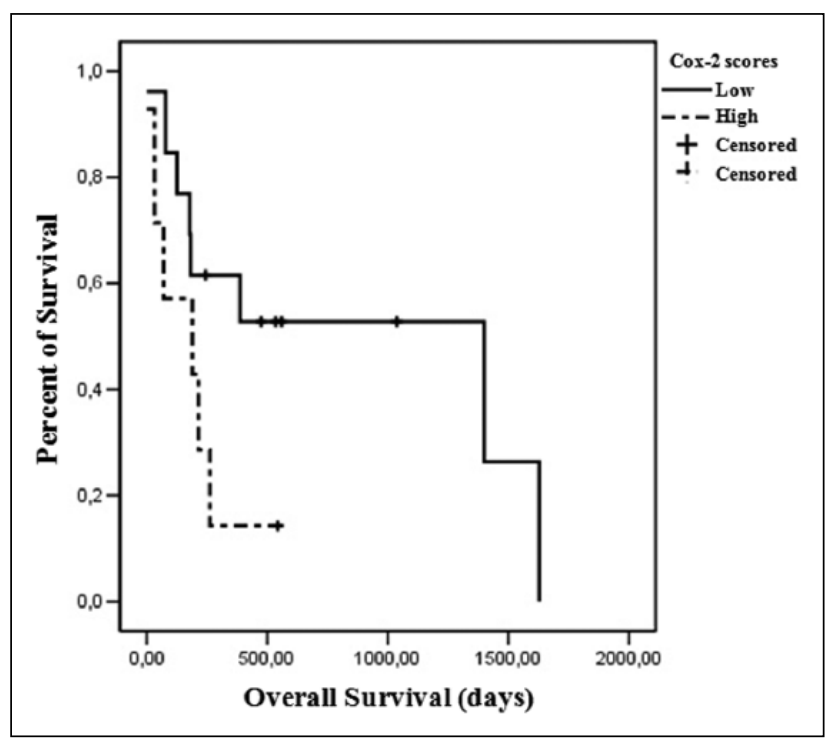

Figure 3 Overall survival curves for 20 queens according to cyclooxygenase (COX)-2 immunohistochemical staining. Thirteen cats had low COX-2 score neoplasms and seven had high COX-2 score neoplasms $(P=0.089)$

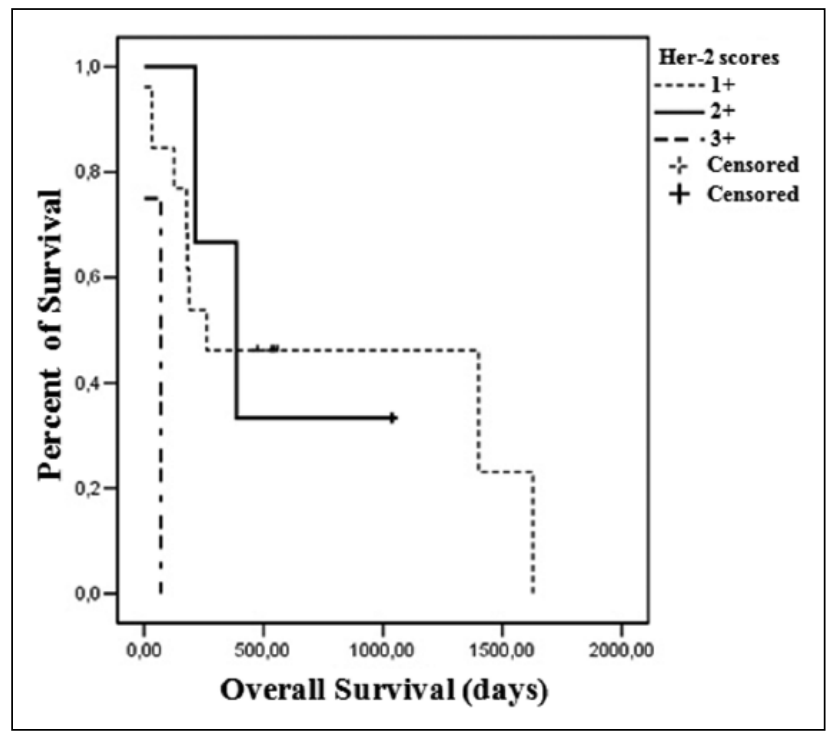

Figure 4 Overall survival curves for 18 queens according to human epidermal growth factor receptor type 2 (HER-2) immunohistochemical staining. Thirteen cats had HER2 neoplasms with a score of $1+$; three cats had HER-2 neoplasms with a score of $2+$; and two cats had HER-2 neoplasms with a score of $3+$ neoplasms $(P=0.012)$

methodology standardization for HER-2 expression could explain the discrepancy between our findings of $10 \%$ HER-2 overexpression in primary tumors and the 57\% and $5.5 \%$ in the other studies, which considered scores of $2+$ and $3+$ as overexpression. ${ }^{29,31,37}$ As described in human breast cancer, ${ }^{38}$ prior feline studies and this study, ${ }^{37}$ higher HER-2 scores were more frequently expressed in

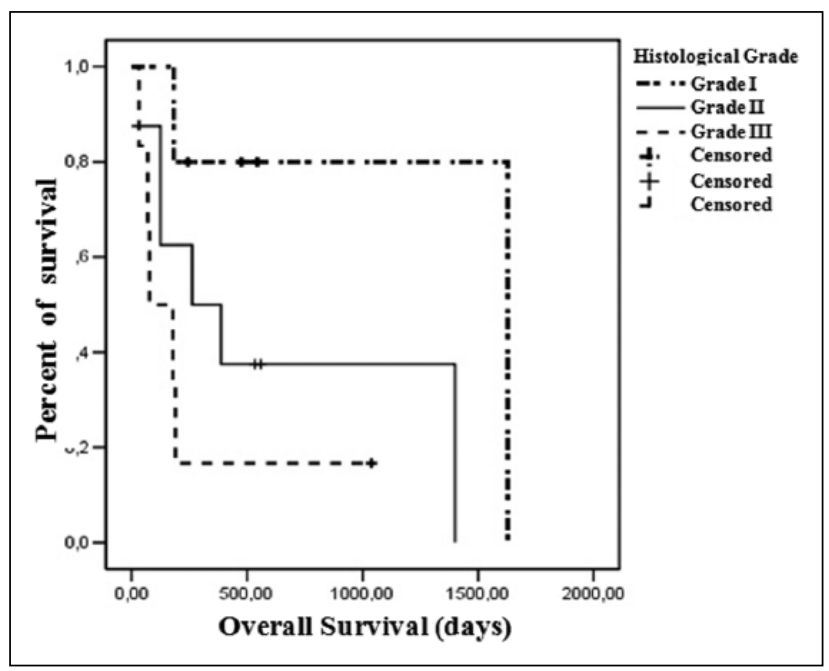

Figure 5 Overall survival curves for 19 female cats according to histologic grade. Five queens had grade I neoplasms; eight had grade II neoplasms; and six had grade III neoplasms $(P=0.080)$

ER-negative tumors. Contradicting previous studies in both humans and feline mammary tumors, $37,39,40$ HER-2 expression was higher in smaller tumors and this indicates that epidermal growth factor stimulation may play a role in the early stages of carcinogenesis. The prognostic significance of HER-2 expression was supported by a previous study, ${ }^{29}$ and this study by the association of OS with HER-2 immunohistochemical status.

COX-2 is a rapidly inducible enzyme that is involved with malignant transformation and tumor progression by affecting cell proliferation, mitosis, cell adhesion, apoptosis, angiogenesis and immune surveillance. ${ }^{30,41}$ Prior studies have reported high positivity $(87 \%$ and $95 \%$ ) in MFMGN, ${ }^{30,31}$ and this study also demonstrated high COX-2 staining, which indicates that COX-2 may have a role in feline mammary gland neoplasm carcinogenesis. ${ }^{42}$ To our knowledge this is the first study to compare COX-2 expression in both primary and metastatic tumors. Although, in a previous study no clear advantage was demonstrated in treating MFMGN with COX2,12 COX-2 could represent a potential target for therapeutic strategies with COX-2 inhibitors, ${ }^{42}$ and animals presenting metastasis and high COX-2 scores could potentially present a more significant clinical benefit. Elevated COX-2 expression correlated with a poor prognosis, as demonstrated by the differences in OS in this and previous studies. ${ }^{30}$

Our study also demonstrated a predominance of score 2 immunostaining for VEGF expression in both primary and metastatic tumors, but there was no difference in OS that correlated with this expression, even though VEGF is a potent angiogenic factor involved in tumor growth, invasion and metastasis..$^{926,20}$ 


\section{Conclusions}

Aggressive surgical excision remains the main treatment option for MFMGN, and additional therapeutic interventions are necessary. The study of prognostic factors revealed that some of these factors might be considered predictive, with treatments involving the inhibition of ovarian hormones and COX-2 possibly representing therapeutic options for MFMGN. When evaluating disease progression, COX-2 scores and the Ki-67 index should be analyzed in primary tumors and metastases. Histologic grade, HER-2 overexpression and COX-2 scores were found to influence the OS of queens directly. Studies involving MFMGN should employ similar and strict methodologies to enable data comparison.

Conflict of interest The authors declared no potential conflicts of interest with respect to the research, authorship, and/ or publication of this article.

Funding This research was supported by the Fundação de Amparo à Pesquisa do Estado de São Paulo (FAPESP), Coordenacão de Aperfeiçoamento de Pessoal de Nível Superior (CAPES), Conselho Nacional de Desenvolvimento e Pesquisa $(\mathrm{CNPq})$ and Fundação de Amparo à Pesquisa do Estado de Minas Gerais (FAPEMIG).

\section{References}

1 Macewen EG, Hayes AA, Harvey HJ, et al. Prognostic factors for feline mammary tumours. J Am Vet Med Assoc 1984; 185: 201-204.

2 Misdorp W. Tumors of the mammary gland. In: Meuten DJ (ed). Tumors in domestic animals. 4th ed. Ames, IA: Iowa State Press, 2002, pp 575-606.

3 Overley B, Shofer FS, Goldschmidt MH, et al. Association between ovariohysterectomy and feline mammary carcinoma. J Vet Intern Med 2005; 19: 560-563.

4 Lana SE, Rutteman GR and Withrow SJ. Tumors of the mamary gland. In: Withrow FJ and Vail DM (eds). Withrow \& MacEwen's small animal clinical oncology. 4th ed. Philadelphia, PA: WB Saunders, 2007, pp 619-636.

5 Bostock DE. Canine and feline mammary neoplasms. $\mathrm{Br}$ Vet J 1986; 142: 506-515.

6 Misdorp W, Else RW, Hellmén E, et al. Histological classification of mammary tumors of the dog and the cat. 2nd Series. Vol VII. Washington DC: Armed Forces Institute of Pathology, American Registry of Pathology and the World Health Organization Collaborating Center for Worldwide Reference on Comparative Oncology, 1999, pp 1-59.

7 Allred CD, Harvey JM, Bernardo M, et al. Prognostic and predictive factors in breast cancer by immunohistochemical analysis. Mod Pathol 1998; 11: 155-168.

8 Zappulli V, Rasotto R, Caliari D, et al. Prognostic evaluation of feline mammary carcinomas a review of the literature. Vet Pathol 2015; 52: 46-60.

9 Giménez F, Hecht S, Craig LE, et al. Early detection, aggressive therapy: optimizing the management of feline mammary masses. J Feline Med Surg 2010; 12: 214-224.
10 Novosad CA, Bergman PJ, O'Brien MG, et al. Retrospective evaluation of adjunctive doxorubicin for the treatment of feline mammary gland adenocarcinoma: 67 cases. $\mathrm{J} \mathrm{Am}$ Anim Hosp Assoc 2006; 42: 110-120.

11 Hughes K and Dobson JM. Prognostic histopathological and molecular markers in feline mammary neoplasia. Vet J 2012; 194: 19-26.

12 Borrego JF, Cartagena JC and Engel J. Treatment of feline mammary tumors using chemotherapy, surgery and a COX-2 inhibitor drug (meloxicam): a retrospective study of 23 cases (2002-2007). Vet Comp Oncol 2009; 7: 213-221.

13 Mcneill CJ, Sorenmo KU, Shofer FS, et al. Evaluation of adjuvant doxorubicin-based chemotherapy for the treatment of feline mammary carcinoma. J Vet Intern Med 2009; 23: $123-129$.

14 Cassali GD, Lavalle GE, Ferreira E, et al. Consensus for the diagnosis, prognosis and treatment of canine mammary tumors-2013. Brazilian J Vet Pathol 2014; 7: 38-69.

15 Elston CW and Ellis IO. Assessment of histological grade. In: Elston CW and Ellis IO (eds). The breast. 3rd ed. London: Churchill Livingstone, 1998, pp 365-384.

16 Dutra AP, Granja NVM, Schmitt FC, et al. C-erbB-2 expression and nuclear pleomorphism in canine mammary tumors. Braz J Med Biol Res 2004; 37: 1673-1681.

17 Hammond MEH, Hayes DF, Dowsett M, et al. American Society of Clinical Oncology/College of American Pathologists guideline recommendations for immunohistochemical testing of estrogen and progesterone receptors in breast cancer. Arch Pathol Lab Med 2010; 134: $48-72$.

18 Wolff AC, Hammond ME, Schwartz JN, et al. American Society of Clinical Oncology/College of American Pathologists guideline recommendations for human epidermal growth factor receptor 2 testing in breast cancer. Arch Pathol Lab Med 2007; 131: 18-43.

19 Lavalle GE, Campos CB, Bertagnolli AC, et al. Canine malignant mammary gland neoplasms with advanced clinical staging treated with carboplatin and cyclooxygenase inhibitors. In Vivo 2012; 26: 375-379.

20 Tsai H, Yang I, Lin C, et al. Predictive value of vascular endothelial growth factor overexpression in early relapse of colorectal cancer patients after curative resection. Int $J$ Colorectal Dis 2013; 28: 415-424.

21 Taylor R. Interpretation of the correlation coefficient: a basic review. J Diagn Med Sonograph 1990; 6: 35-39.

22 Mills SW, Musil KM, Davies JL, et al. Prognostic value of histologic grading for feline mammary carcinoma: a retrospective survival analysis. Vet Pathol 2015; 52: 238-249.

23 Seixas F, Palmeira C, Pires MA, et al. Grade is an independent prognostic factor for feline mammary carcinomas: a clinicopathological and survival analysis. Vet J 2011; 187: 65-71.

24 Castagnaro M, Casalone C, Bozzetta E, et al. Tumour grading and the one-year post-surgical prognosis in feline mammary carcinomas. J Comp Pathol 1998; 119: 263-275.

25 Millanta F, Lazzeri G, Mazzei M, et al. MIB-1 labeling index in feline dysplastic and neoplastic mammary lesions and its relationship with postsurgical prognosis. Vet Pathol 2002, 39: 120-126. 
26 Millanta F, Lazzeri G, Mazzei M, et al. Correlation of vascular endothelial growth factor expression to overall survival in feline invasive mammary carcinomas. Vet Pathol 2002; 39: 690-696.

27 Dias Pereira P, Carvalheira J and Gartner F. Cell proliferation in feline normal, hyperplastic and neoplastic mammary tissue - an immunohistochemical study. Vet J 2004; 168: 180-185.

28 Millanta F, Calandrella M, Bari G, et al. Comparison of steroid receptor expression in normal, dysplastic, and neoplastic canine and feline mammary tissues. Res Vet Sci 2005; 79: 225-232.

29 Millanta F, Calandrella M, Citi S, et al. Overexpression of HER-2 in feline invasive mammary carcinomas: an immunohistochemical survey and evaluation of its prognostic potential. Vet Pathol 2005; 42: 30-34.

30 Millanta F, Citi S, Della Santa D, et al. Cox-2 expression in canine and feline invasive mammary carcinomas: correlation with clinicopathological features and prognostic molecular markers. Breast Cancer Res Treat 2006; 98: 115-120.

31 Rasotto R, Caliari D, Castagnaro M, et al. An immunohistochemical study of HER-2 expression in feline mammary tumours. J Comp Pathol 2011; 144: 170-179.

32 Las Mulas JM, Niel MV, Millán Y, et al. Immunohistochemical analysis of estrogen receptors in feline mammary gland benign and malignant lesions: comparison with biochemical assay. Domest Anim Endocrinol 2000; 18: 111-125.

33 Las Mulas JM, Niel MV, Millán Y, et al. Progesterone receptors in normal, dysplastic and tumourous feline mammary glands. Comparison with oestrogen receptors status. Res Vet Sci 2002; 72: 153-161.
34 Hayden DW, Barnes DM and Johnson KH. Morphologic changes in the mammary gland of megestrol acetatetreated and untreated cats: a retrospective study. Vet Pathol 1989; 26: 104-113

35 Goldhirsch A, Glick JH, Gelber RD, et al. Meeting highlights: international expert consensus on the primary therapy of early breast cancer. Ann Oncol 2005; 16: 1569-1583.

36 Eccles SA. The role of c-erbB-2/HER2/neu in breast cancer progression and metastasis. J Mammary Gland Biol Neoplasia 2001; 6: 393-406.

37 Ordás J, Millán Y, Dios R, et al. Proto-oncogene HER-2 in normal, dysplastic and tumorous feline mammary glands: an immunohistochemical and chromogenic in situ hybridization study. BMC Cancer 2007; 7: 179-184.

38 Quénel N, Waffiart J, Bonichon F, et al. The prognostic value of c-erbB2 in primary breast carcinomas: a study on 942 cases. Breast Cancer Res Treat 1995; 35: 283-291.

39 Swede H, Moysich KB, Winston JD, et al. Variation of the prognostic significance of HER-2 expression in breast cancer according to tumor size. Breast $J$ 2003; 9: 98-105.

40 Azizun-Nisa, Bhurgri Y, Raza F, et al. Comparison of ER, PR and HER-2/neu (c-erb $B$ 2) reactivity pattern with histologic grade, tumor size and lymph node status in breast cancer. Asian Pac J Cancer Prev 2008; 9: 553-556.

41 Williams CS, Mann M and Dubois RN. The role of cyclooxygenases in inflammation, cancer, and development. Oncogene 1999; 18: 7908-7916.

42 Sayasith K, Sirois J and Doré M. Molecular characterization of feline Cox-2 and expression in feline mammary carcinomas. Vet Pathol 2009; 46: 423-429. 